\title{
In-situ Droplet Inspection and Closed-Loop Control System using Machine Learning for Liquid Metal Jet Printing
}

\author{
Tianjiao Wang ${ }^{1}$, Tsz-Ho Kwok ${ }^{2 *}$, Chi Zhou ${ }^{1}$, and Scott Vader $^{3}$ \\ ${ }^{1}$ Department of Industrial and Systems Engineering, \\ University at Buffalo, the State University of New York, Buffalo, NY 14260, U.S.A \\ ${ }^{2}$ Department of Mechanical and Industrial Engineering, \\ Concordia University, Montreal, QC H3G 1M8, Canada \\ ${ }^{3}$ Vader Systems, 385 Crosspoint Parkway, Suite 104, Getzville, NY 14068, U.S.A \\ twang38@buffalo.edu,tszho.kwok@concordia.ca,chizhou@buffalo.edu, scott@vadersys.com
}

\begin{abstract}
Liquid Metal Jet Printing (LMJP) is a revolutionary 3D printing technique in fast but low-cost additive manufacturing. The driving force is produced by magneto-hydrodynamic property of liquid metal in an alternating magnetic field. Due to its integrated melting and ink-jetting process, it can achieve 10x faster speed at $1 / 10^{\text {th }}$ of the cost as compared to current metal 3D printing techniques. However, the jetting process is influenced by many uncertain factors, which impose a significant challenge to its process stability and product quality. To address this challenge, we present a closed-loop control framework by seamlessly integrating vision-based technique and neural network tool to inspect droplet behaviours and accordingly stabilize the printing process. This system automatically tunes the drive voltage applied to compensate the uncertain influence based on vision inspection result. To realize this, we first extract multiple features and properties from images to capture the droplet behaviour. Second, we use a neural network together with PID control process to determine how the drive voltage should be adjusted. We test this system on a piezoelectric-based ink-jetting emulator, which has a very similar jetting mechanism to the LMJP. Results show that significantly more stable jetting behaviour can be obtained in real-time. This system can also be applied to other droplet related applications owing to its universally applicable characteristics.
\end{abstract}

Keywords: Additive manufacturing, metal jetting, process inspection, closed-loop control, vision, neural network

\section{Introduction}

\subsection{Background}

Additive manufacturing (AM) or 3D printing has been hailed as the third industrial revolution in the unique way that products are designed and manufactured [1]. Due to the elegant concept of the layer by layer fabrication, AM can build complex objects with a wide variety of materials and functions. This opens up tremendous opportunities for a wide range of applications including aerospace, automotive, defence, and biomedical industries [2]. With the advancement of material, machine, and process, metal 3D printing is now the fastest growing segment among 3D printing technologies [3]. However, most of the current metal 3D printing applications involve high cost and

* Corresponding author. 
low-speed metal powder sintering or melting [4-7]. Recently, a revolutionary liquid metal jet printing (LMJP) alternative $[8,9]$ has been explored and recognized as a promising emerging process that can drastically lower manufacturing part costs while doubling existing printing speed. This game-changing technology is opening unprecedented opportunities in advanced manufacturing.

Vader Systems, a startup company in Buffalo, NY, is developing and commercializing the world's first molten metal 3D printer using proprietary LMJP technology based on magneto-hydrodynamic inkjet printing process [8]. The LMJP technology patterns magneto-hydrodynamic liquid metal into complex $3 \mathrm{D}$ parts $10 x$ faster at $\mathbf{1} / \mathbf{1 0} \mathbf{t h}^{\text {th }}$ of the part cost as compared to current methods [8]. This includes the earth's most abundant metal Aluminum, which has been widely used in missioncritical heavy industries, yet extremely challenging to handle by other metal printing technologies. The molten solid metal in LMJP rather than sintered

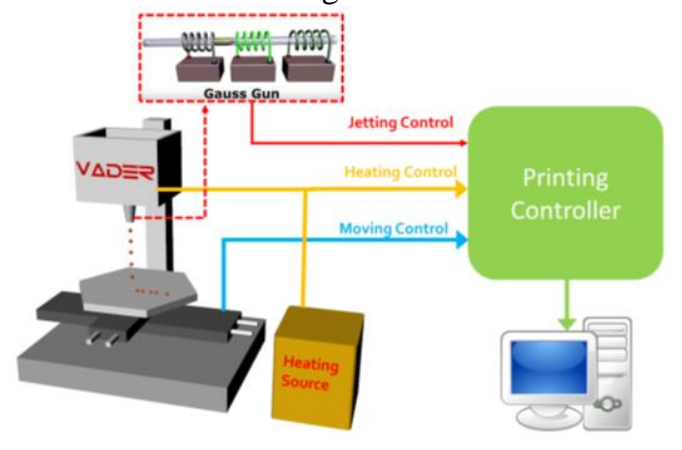

Figure 1: Liquid metal jet printing system powder leads to dense metal parts with much finer micro-structure that have $30 \%$ or greater increase in ultimate tensile strength [11]. The main structure of this system is shown in Figure 1.

Though tremendous efforts and progress have been made in the LMJP process during the past few years, as a brand-new technology, there are still multiple challenges such as the limited choice of material, e.g., has to be conductive or pre-charged, low melting point $\left(660^{\circ} \mathrm{C}\right)$, and the difficulty in controlling the wetting property and coalescence behavior of the jetted metal droplet, which handicap its large-scale commercialization in practice. One of the major challenges is that LMJP process suffers from low process reliability and product quality issues. Physics-based modeling approaches have been proposed to predict the process drift and suggest corrective actions. However, the complex printing process (energy-matter interaction, phase changing, thermal-mechanical interaction) and the limitation of current computational tools hinders its practical applications in 3D printing processes $[12,13]$. This gap has been reported in recent additive manufacturing roadmap reports by both the government agencies and industrial stockholders [14-17]. Given the layer-by-layer nature of 3D printing, if the process drifts are not corrected in a timely manner, defects will propagate into subsequent layers, and thus deleteriously affect the function integrity (fatigue, strength, geometric integrity) of the part. Currently, the metal 3D printing systems are in an open-loop configuration, and the measurement of part quality is done offline, leading to material and energy waste and even devastatingly affect the structural health conditions and infrastructural integrity of many important engineering systems, especially for mission-critical applications such as aerospace, defence, and automobile areas. In-situ process-monitoring and process-control are promising to address this challenge. To fill in this research gap and advance the technology development, we develop and validate a novel closed-loop control system which has a vision-based droplet inspection and a neural network based proportional-integralderivative (PID) technology. Specifically, droplet formation is one of the most important factors associated with the printing quality and reliability in the inkjet metal 3D printing process. It is vital to on-line monitor and in-situ control the jetting behavior including the droplet volume, speed, and location in jetting history, which would affect the geometrical and functional integrity of the printed part. The aim of this paper is to design and verify a sensing and detection module that can capture high-fidelity data of the droplet and extract critical information for the downstream decision making for in-situ correction, and ultimately improve the process reliability, reproducibility, and printing quality of LMJP process. The result from this paper will be a feedback control system that continuously monitors the pattern of the droplets in the LMJP process using the stroboscopic imaging technique and adjusts the applied voltage level to compensate the difference between the observed pattern and the desired pattern due to environmental changes and unexpected events. We acknowledge 
that these individual techniques (image processing, neural network, and closed-loop control) are well established and widely used in various applications. However, to the best of our knowledge, we for the first time seamlessly integrate these techniques by taking advantage of the unique merits of each technique to effectively solve the pressing problem encountered in 3D printing. We believe the research outcome opens up a new avenue for the research in the quality control area in additive manufacturing and other advanced manufacturing domain. More specifically, the integration and interaction between the key techniques provide valuable guidance to the researchers to explore new means to solve the process control and in-situ quality certification problems, which have been proven a grand challenge in the past years.

\subsection{Related work}

Image- and video-based approaches have been widely used for monitoring the $3 \mathrm{D}$ printing process to improve the printing quality. Mazumder [18] used cameras to monitor the height of metal deposition in a laser cladding 3D printing system, through which the layer height can be controlled and better printing quality can be obtained. However, this technology can only control the dimension in the vertical direction of the printed part, and the quality improvement is rather limited. $\mathrm{Hu}$ [19] built a closed-loop control system for the heat input based on infrared images of the molten pool in a laser-based additive manufacturing system. Toyserkani [20] developed a pattern recognition algorithm to obtain the clad's height and angle, and a PID controller is developed based on that. The results show an effective improvement of the geometrical integrity. Salehi [21] developed a PID closed-loop controller on LabVIEW to control the temperature of the melt pool, but the results show that controlling temperature alone cannot produce expected quality improvement due to the complexity of the laser cladding process. Faes [22] demonstrated a way to use laser scan to monitor the printed part shape on an extrusion based 3D printer prototype, but the application of this technique is limited to extrusion-based 3D printing, and the measurement error is related to the printing materials due to its interaction with the laser. Cheng [23] presented a closed-loop online system where the feedback is obtained from 3D images, and a fuzzy controller is developed to fulfill the process. Regarding vision and image processing for studying droplet behavior, there are different image processing technologies developed in different areas. Hijazi [24] used cameras to detect the small droplet from a spray nozzle to quantify the process in precision agriculture, and shape matching and contour tracking are used to detect the droplet. However, the high-speed camera is required for precision monitoring and detection, which limits its wide application in cost sensitive area. Pfeifer et al. [25] used camera coupled with pulse laser to characterize the fuel droplet from a spray system in high-pressure conditions. The droplet velocity, size, and spatial distribution are particularly studied. Kwon [26] used the camera to detect the droplet speed of ink printing. By image processing, Cabezas [27] shows that the surface tension of the liquid material can be measured. Blaisot [28] applied image processing to identify droplet size and morphology, which can be used to analyze the diesel spray behavior. Kwon [26] demonstrated that by using edge detection techniques, the speed of ink droplet can be measured, and this technology can be used for in-situ measurement. For the stereolithography-based 3D printing process, Xu et al. used a thermal camera to study the shape deformation [29] and also used a 3D scanner-based close loop framework for shape deformation control [30]. The research works reviewed above show that the image processing is promising for droplet characteristic analysis. However, the in-situ closed-loop control is still missing due to complex and dynamic droplet formation and propagation process. This paper proposes a holistic framework that seamlessly integrates online monitoring by image processing technique and in-situ closed-loop control module to effectively detect and subsequently correct the process drift and anomalies toward high-quality metal 3D printing. 


\section{Setup and Framework}

In LMJP process, the metal filament is melted by a resistive heater, and the liquefied metal is then propelled by alternative inductive force in a drop-on-demand manner. In this research, we first prototype a piezo inkjet printing emulator which applies the same drop-on-demand principle as LMJP, and develop a vision system to study the in-situ detection and correction problem.

\subsection{Piezo inkjet printing emulator}

The jetting force in LMJP system is produced by magnetic field constructed by a coil around the melted metal, and the current in the coil changes and creates a time-varying magnetic field. When the liquid metal is exposed in a time-varying magnetic field, eddy currents are induced in the metal and create a repulsive magnetic field which pulls the liquid metal apart from the coil. When enough liquid metal is pushed out from the nozzle, a droplet will be formed and ejected caused by Rayleigh instability [31, 32]. The print head structure is shown in Figure 2(a). LMJP has proven to be a feasible and promising metal 3D printing technology, and complex parts have been successfully printed in current LMJP prototype machine. However, as an emerging technology, LMJP is still in early R\&D stage, and the accessibility to the prototype machine is very limited. Because LMJP is based on the same drop-on-demand mechanism as the piezo-driven inkjet printing which is widely used in 2D printing and 3D printing industries, in this paper we use a piezo-based ink jet device as an emulator to study the proposed online monitoring and in-situ correction system. Specifically, the piezo-driven micro-inkjet device is used in this work. Its main component is a glass tube covered by piezoelectric (PZT) material with a pair of inner and outer electrodes. When a differential voltage is applied to the electrode pair, the tubular PZT expands and squeezes the glass tube. This process generates an extrusion force to move the liquid material out of the glass tube, and then produces droplets. Its structure is shown in Figure 2(c). Zhou et al. developed a simulator for the control of droplet jetting in the piezo-based multi-jet modeling process [33]. The processes of these two devices are very similar, and they both use electricity to generate extrusion force. By controlling the voltage or the current, they can achieve different jetting behavior of the liquid material, and both processes use trapezoid waveform to drive the device. In addition, the jetting behavior (droplet initiation and propagation) in Figure 2(b) and Figure 2(d) show that the two processes have very similar jetting properties. Furthermore, several researchers in the early 1990's investigated liquid metal jetting process for both printing and spherical balls fabrication applications. In these early studies, the same drop-on-demand printing mechanism and similar jetting behavior between LMJP and piezo-driven inkjet printing were demonstrated [10, 34-39]. Based on this similarity, we argue that the developed monitoring and control system can be readily deployed in the LMJP metal printer once it is accessible. Improving the process reliability and part quality for the LMJP process using the proposed monitoring and correction approach is left for our future work. 


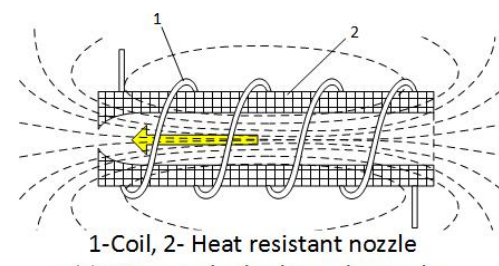

(a) Magneto-hydrodynamic nozzle

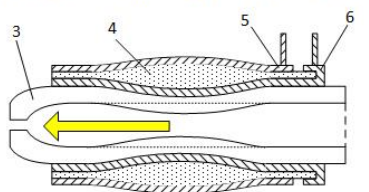

3-Glass tube, 4-Piezoelectric material, 5-Outer electrode, 6-Inner electrode (c) Piezo nozzle

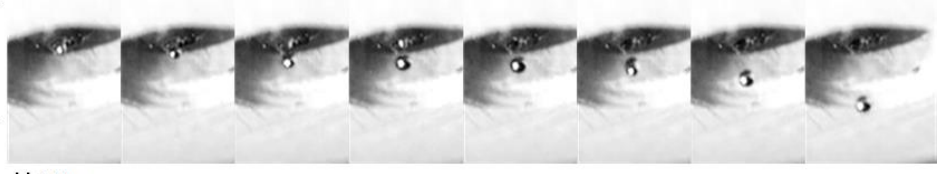

(b) Magneto-hydrodynamic jetting

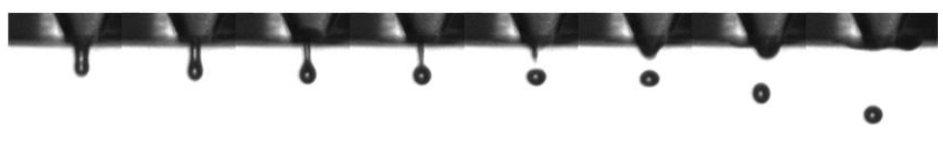

ப $100 \mu \mathrm{m}$

(d) Piezo nozzle jetting

Figure 2: The LMJP system (a, b [8]) compared with the 3D printing emulator of piezoelectric model (c, d).

\subsection{Hardware setup}

The LMJP process is a powerful but a relatively complex process. For a complete understanding of the process, it would be helpful to formulate a mathematical model to study the jetting process and predicate the printing behavior and the characteristics of the fabricated parts. However, for LMJP process, it is very challenging if not impossible to formulate such model, due to the nature of the process complexity. Direct measurement of the quality criteria during the building process is also not possible, therefore an indirect measurement becomes promising to study and predict the complex jetting process. The patterns of droplets jetted by the nozzle are such indirect measurements and contain several characteristics that can serve as features to indicate the process state. The crucial point is to identify those characteristics that serve as good features for process and part quality. These features are measured from the droplet patterns in a first step, and in a second step they are used for quality evaluation and to decide how to respond to observed defects in the process.

We develop a vision system on a piezo-based emulator as shown in Figure 3. The emulator system includes a piezo dispenser, piezo driver, computer, CCD camera (STC-MB33USB, Sensor Technologies), and strobing LED. The piezo and strobing LED is controlled by the piezo driver. The piezo driver generates two digital pulses, where the first one is used as a trigger signal to generate the trapezoid waveform and the second one is used to control the strobing LED light. The second pulse is synchronized with the first pulse, and the delay time between the first and the second pulse is adjusted so that the images can be captured at certain desired time instants. As the LED light and the CCD camera are triggered together to shine and capture at the same time, the jetting images appear to be frozen and clear. Therefore, we can apply image processing and analysis to the jetting images, and the droplet patterns can be studied and understood. When we extend the delay time between these two pulses, the image will show the droplet of a later time after dropping. With a continuously swept delay time, dynamic droplet behavior can be obtained from a sequence of images correspondingly. It is remarked that the print head and the camera is fixed in the setup, but the printing platform is moving, so that the droplet properties will not be affected by the movement of the print head. 


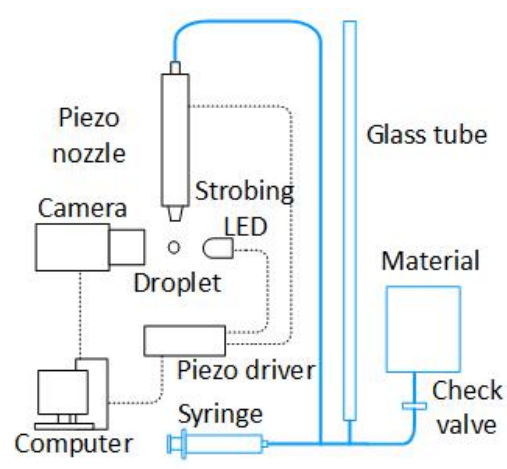

(a) Setup schematic diagram

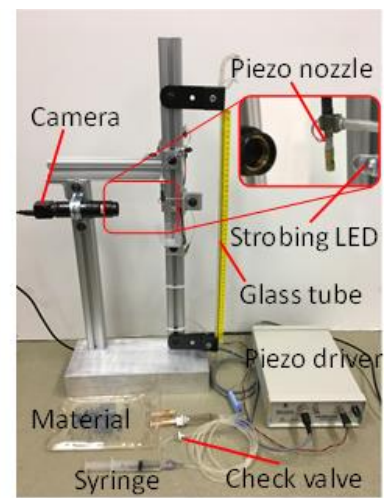

(b) Setup picture

Figure 3: Hardware setup of the piezo-based LMJP emulator.

In a later experiment, a pressure adjustment system is used to simulate the pressure change applied to the printing material, which is realized by a syringe and a glass tube. By changing the position of the cylinder in the syringe, the liquid material will be pumped into the glass tube. The liquid level in the glass tube will reflect the pressure applied to the printing material. The changing pressure acts as an uncontrollable external variable.

\subsection{Framework overview}

The jetting behaviour depends on many features of the whole system including the drive voltage applied, orifice size of the nozzle, external pressure, liquid viscosity, surface tension, etc. To control the manufacturing process to minimize defects, we first classify the process parameters into two categories: "random" and "assignable". Some defects are of assignable causes, such as inappropriate parameter settings, which are of repetitive nature unless some corrective actions are taken. Other defects are random disturbances or uncontrollable effects, e.g., the external pressure decreases with the consumption of the materials; for the aqueous colloidal suspension materials, the viscosity and density may change during the printing process due to local aggregation. These changing parameters make it very difficult to obtain stable jetting behaviour. The purpose of process control is to adjust the controllable parameters to minimize the predicted defects (assignable causes) and also to compensate the random defects in subsequent processes. Specifically, the pattern of the droplet is essential for the creation of defects, we need to adjust process parameters to create appropriate droplets and thus achieve better product quality. For the uncontrollable effects that cannot be compensated by parameter adjustment and can only be removed by hardware modification based on the flashing synchronous image capture mechanism, they should be understood and should not mislead the process control. Several factors could affect the pattern of the droplet, including material, drive voltage, frequency, temperature, pressure, and distance between the nozzle and the surface. Among all the parameters, the drive voltage is the key, as it is applicable to different materials over different nozzle size and has much smaller inertia than air pressure that gives shorter reaction time, which is critical in the online control system. We will focus on voltage control in this paper, but the same approach can be extended to other parameters once they can be controlled reliably and accurately by the precision control system, such as pressure/vacuum using the pneumatic controller.

In this paper, we build a novel closed-loop controller which integrates image processing and neural network technology. As the ink jetting process is very complex and may be influenced by multiple parameters, where most of these influences are non-linear, the major challenge is how to use the captured images from the vision system to make decisions on adjusting piezo drive voltage. As the first step toward the corrective decision making, we formulate a neural network model to establish the complex relation between the controllable variable and the droplet features. By inferring the functions 
of the network from labeled training data, each of which is a pair consisting of an input vector (droplet features) and an output value (voltage level), the system can map the jetting behaviour to a corresponding value of the controllable variable. In this way, simple control strategy like PID process can be used to compare the jetting behaviour to the target point to address the complex non-linear control problem in an on-line and in-situ manner without human interaction and specific expertise, which otherwise would be extremely challenging for traditional model-driven controller design. The structure of the system is shown in Figure 4. The reference input is the desired jetting behaviour. In this case, the ideal jetting behaviour is that each pulse of the input signal only generates one single droplet with sufficient volume, and without satellite following behind it. The image sequence of the droplets captured by CCD camera is fed back to the controller, and then the droplet features are extracted by image processing. By mapping these features into a virtual voltage value using the neural network, and then comparing with the target features, a PID process is used to adjust the drive voltage, which is then applied to the piezo driver. Such iterative process is the essential components of the closed-loop control system to achieve more stable and reliable jetting behaviour.

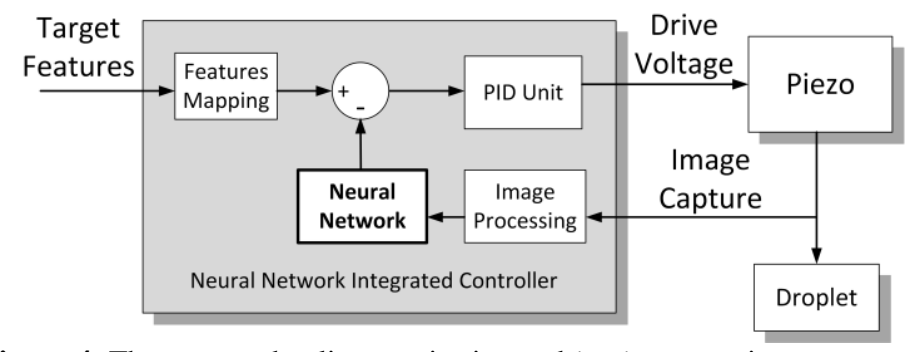

Figure 4: The proposed online monitoring and in-situ correction system

\section{Vision-based Droplet Features Extraction}

The goal of the vision system is to capture the droplet patterns and extract some features of the droplets from the images. Typical features from inkjet techniques [26, 40] can be used, e.g., satellite, ligament, speed, volume, which can be measured in real time. Among these features, the volume and ligament convey the geometric information of the droplet, the satellite represents its morphologic status and the speed infers its kinematical behavior. Although the droplet patterns obtained from 2D images can reveal the quality characteristics, a single feature is not sufficient to determine the process state, and we have to utilize more features to distinguish between inappropriate parameter settings and random defects and thus increase the sensitivity and accuracy of the inspection system. For instance, the effect of the drive voltage on the droplet size and velocity has been studied in Li's thesis [41]. The research shows that when the amplitude of the drive voltage increases, both of the droplet volume and the velocity will increase. This can be explained by volume changes of the piezo due to the piezoelectric effect: $\frac{\Delta V_{p}}{V_{p}}=-d_{31} \frac{U}{t}$, where $V_{\mathrm{p}}$ is the volume of the piezoelectric actuator, $d_{31}$ is the piezoelectric strain constant, $U$ is the drive voltage and $t$ is the thickness of the piezoelectric tube. From this equation, we can understand that a higher voltage can lead to a larger volume change of the piezoelectric actuator, as it is attached to the glass tube. The glass tube will be squeezed simultaneously, and this will cause more liquid ejected. Similarly, larger induced acoustic waves will generate a higher velocity of the droplet. Therefore, one can calculate the velocity of a droplet and find out if the voltage is optimal. However, it is far more complicated in practice, and the same phenomenon may be caused by different assignable factors and random disturbances. One example is that the abnormal speed of droplet in Figure 5(a) is caused by the instability of back pressure, which is an uncontrollable random deflect but not because of the voltage. Another example is shown in Figure $5(\mathrm{c})$, the number of satellite can be increased because of a low voltage and thus low deposition rate 
that the volume is not enough to form a sharp drop, or a high voltage and thus high deposition rate that the volume is too much to form a single drop.

(a) Uncontrollable random deflect

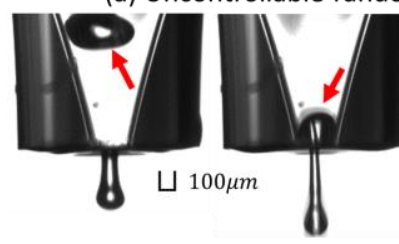

$t=114 \mu s$

$154 \mu s$

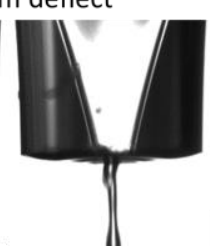

(b) Droplet dynamics

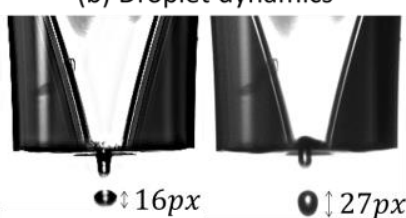

(⿶) $16 p x$

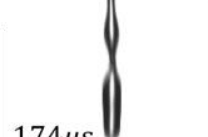

$174 \mu \mathrm{s}$ $t=194 \mu s$

$234 \mu \mathrm{s}$

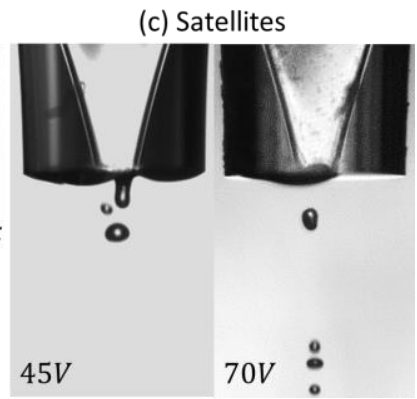

Figure 5: (a) The speed of droplet is increased by the instability of vacuum. (b) The detected volume of droplet is kept changing. (c) The number of satellites can be increased because of the voltage is too low or too high.

Because the droplet is subjected to fluid dynamics, the same droplet at different time instances may appear to be having different volume as shown in Figure 5(b). To incorporate the droplet dynamics in the system, in this paper we present a solution with a hierarchical scheme for defect detection as shown in Figure 6. Each input image is analyzed individually first, and features from each frozen image are extracted. The extracted features from the images in different time steps are then summarized as properties through a set of rules. After all, the process state is deduced from the properties. The process state here is the voltage level.

\subsection{Feature extraction}

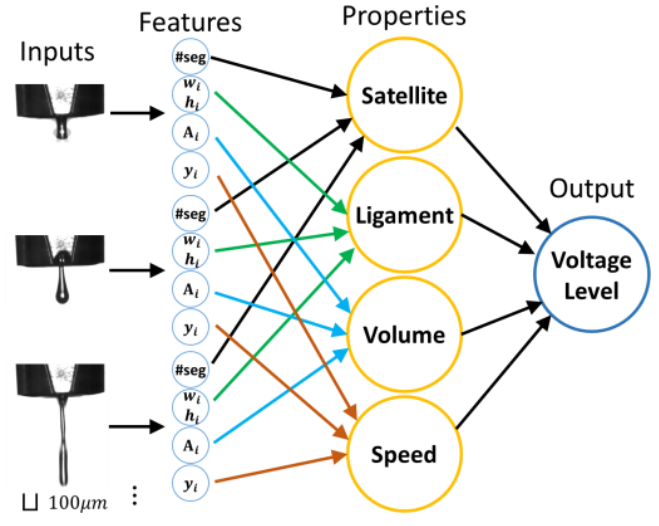

Figure 6: Defect detection architecture

Given a frozen image, we define a region of interest (RoI) right below the nozzle of a jet image as shown in Figure 7, which can be specified manually if the hardware setup is always fixed, or automatically by detecting the position of the nozzle (using the same method for detecting droplets). In general case, the background is clear and has a good enough contrast to the foreground (i.e., droplets), so simple thresholding can segment the RoI into foreground and background easily. However, Otsu's method [42] can be used to choose a threshold more intelligently through a histogram of the RoI. The connected components on the resultant binary image are the droplets or the ligaments, and if there are internal holes surrounded by foreground after segmentation, they are automatically filled. After that, the features can be extracted. The features are the number of connected components (\#seg), the maximum width $\left(\mathrm{w}_{\mathrm{i}}\right)$ and height $\left(\mathrm{h}_{\mathrm{i}}\right)$, area $\left(\mathrm{A}_{\mathrm{i}}\right)$, and the largest position in the $\mathrm{y}$-axis of each component $\left(\mathrm{y}_{\mathrm{i}}\right)$. The subscript $\mathrm{i}$ indicates the features are extracted separately for each of the connected components ordered from top to bottom. All these features can be extracted easily by a simple flood-fill algorithm [43] in each connected region, and can be done in real-time. 


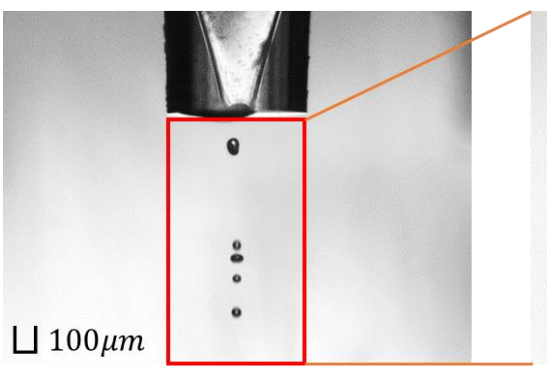

Frozen Image

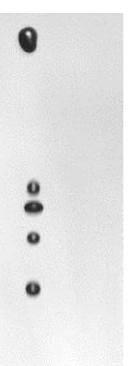

Rol

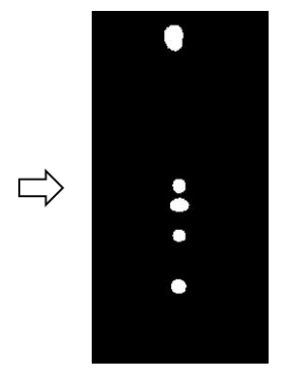

Segmentation

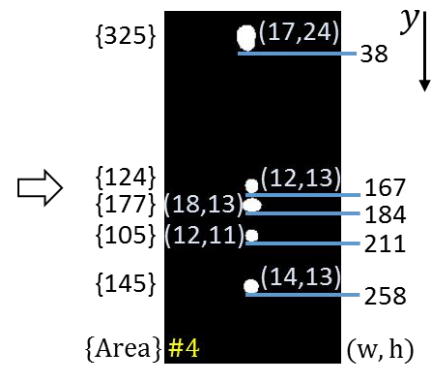

Features

Figure 7: Feature extraction from frozen jet image. Right: \{Area $\}$ represents the area of the extracted droplet, (w, h) represents the width and height of the droplet.

\subsection{Dynamic analysis}

The extracted features from the frozen images are summarized to properties of the droplet pattern. The properties are the satellite, ligament, volume, and speed. Instead of generating a large number of samples to train a system to learn the relationship between the features, the properties, and the voltage level, we define a set of knowledge-based rules by our understanding on the droplet system to deduce the properties from the features, which are simple but effective. Assume there are $\mathrm{m}$ images at different time instants $\left\{\mathrm{t}_{\mathrm{m}}\right\}$ and there are $\mathrm{c}_{\mathrm{t}}\left(\forall \mathrm{t}=\mathrm{t}_{1} \ldots \mathrm{t}_{\mathrm{m}}\right)$ connected components sorted in an order from top to bottom in each image, the rules are defined as follows:

1. The number of satellites is equal to the maximum value of the number of connected components among all frozen images, i.e., Satellite $(\mathrm{N})=\max \left\{\# \operatorname{seg}_{\mathrm{t}}\right\}$. e.g. $\mathrm{N}=5$ as shown in Figure 7.

2. The size of ligament is defined as the maximum value of height-to-width ratio for each connected component among all images, i.e., Ligament $(\mathrm{L})=\max \left\{\mathrm{h}_{\mathrm{i}, \mathrm{t}} / \mathrm{w}_{\mathrm{i}, \mathrm{t}}\right\} .\left(\forall \mathrm{i}=1 \ldots \mathrm{c}_{\mathrm{t}}\right)$. e.g. $\mathrm{L}=24 / 17=1.41$ as shown in Figure 7 .

3. The total volume of a jet is the maximum value of the total area of components among all images, i.e., Volume $(V)=\max \left\{\sum_{i} A_{i, 1}, \sum_{i} A_{i, 2}, \ldots, \sum_{i} A_{i, t}\right\}$. e.g. $V=325+124+177+105+145$ $=876$, as shown in Figure 7.

4. The jetting speed is defined as the travel speed of the lowest droplet, which can be calculated at two different time instants $\left(t_{a}, t_{b}\right)$, i.e., Speed $t_{t}=\frac{p_{t_{b}}-p_{t_{a}}}{t_{b}-t_{a}}$, where $p_{t}$ is the largest value of $y_{i}$ at time $t$, i.e., $\mathrm{y}_{\mathrm{c}_{\mathrm{t}}}$. The jetting speed $(\mathrm{S})$ is the maximum value of Speed $_{\mathrm{t}}$. The $\mathrm{y}_{\mathrm{c}_{\mathrm{t}}}=285$ as shown in Figure 7, as we use strobing, this value can be regarded as a measurement of distance travel from $t=0$ in a simplified case.

The evaluated values in the steps above are also used for screening purpose. For example, when the number of satellites and the jetting speed decrease at time $t$, it is safe to assume that some droplets have left the RoI, and thus the Speed is measured only before $t$; or when there are abnormal changes at time $t$ such as a sudden decrease of satellites $\left(\# \operatorname{seg}_{t} \ll \# s g_{t_{a}}\right)$, sudden increase of ligament size $\left(\right.$ Ligament $_{t} \gg$ Ligament $_{t_{a}}$ ), volume $\left(\right.$ Volume $_{t} \gg$ Volume $_{t_{a}}$ ), or speed $\left(\right.$ Speed $_{t} \gg$ Speed $_{t_{a}}$ ), they are most likely caused by some random disturbances that cannot be controlled (e.g., Figure 5(a)), so this particular droplet pattern is discarded in the control loop. It is worth to mention that the relationships between extracted features and droplet dynamics are linear with one-to-one correspondence, e.g., the speed is computed only by the $y$ coordinate, and the volume is computed only by the area. Therefore, the analysis is efficient yet effective. To analyze the droplet behavior, the imaging system captures images of sequential droplets with a dropping frequency of $300 \mathrm{~Hz}$. The sequential droplets have identical behavior in such a short period. Capturing more images is generally more robust, the 
minimum number of the image required is three according to our algorithm to examine different stages of the jetting process.

\section{Process Control}

The goal of process control is to adjust the drive voltage to minimize the defects caused by assignable or random variables. Gathering the properties of a droplet pattern, they are used to determine the level of voltage for a particular time and environment. The optimal case is that there is only one single droplet with a circular shape, i.e., $\mathrm{N}=1$ and $\mathrm{L}=1$, and it has sufficient volume and speed. For the volume and speed, a pixel-based unit value can be set, e.g. $V=120$ pixels, $S=1$ pixel/ $\mu$ s. Noted that, there is no need of the calibration between the world coordinate and the image coordinate to find the pixel size, because this system focusses on the droplet pattern rather than the exact physics. It is straightforward to determine if a droplet pattern is desired, but it is difficult to define how far from the optimal a droplet is. The properties vary more or less continuously with the voltage level, instead of a well-defined boundary. For example, "if the droplet is fast then the voltage is high". The meanings of the expressions "fast/slow", "large/small volume", or "high/low" do not have exact evaluations and are represented by functions mapping. To account for these dynamics, we employ the neural network to establish the relation between the features and a voltage level. Following the neural network training process, all input values are the features extracted from images in the training data set, and the target data is the drive voltage level under which each image is captured. With this trained neural network, an offset voltage value can be obtained to characterize the jetting behavior based on the image, and then this value can be used to adjust the drive voltage applied on the print head.

\subsection{Neural network training}

To train the neural network, we need to prepare a set of training data, i.e., images of droplet pattern labeled by voltage level. Instead of asking an expert to label the captured images one-by-one, we present a practical procedure to generate the training set. First, as an initialization step, the user tunes the system setting to obtain a good droplet pattern, i.e., $\mathrm{N}=1$ and $\mathrm{L}=1$. In our experiment during the training, the voltage is $45 \mathrm{~V}$, the pressure is $-8 \mathrm{mmH}_{2} \mathrm{O}$, and jetting frequency is $300 \mathrm{~Hz}$. Note that, the values here are just for reference, but the optimal setting varies by time, and it's also why the control system is important. Second, the system captures images and adjusts the drive voltage automatically. The voltage is adjusted step by step with an offset value such as $\pm 1, \pm 2, \pm 3, \ldots, \pm 10$, and this value is used to label the corresponding captured image, which is the output of the network. Third, the feature extraction method is used to extract the four features from the images including the number of satellite, ligament, volume and speed of the droplet, which are used as the inputs of the network. This procedure is repeated for several times to minimize the effect of inaccurate initial setup. We have collected 800 sets of data, in which $70 \%$ of them are used for training, $15 \%$ are used for validation and another $15 \%$ are used for testing. They are used to train a two-layer feed-forward network with ten sigmoid hidden neurons and linear output neurons, using the Levenberg-Marquardt backpropagation algorithm. The trained networks performance and error histogram are shown in Figure 8. 

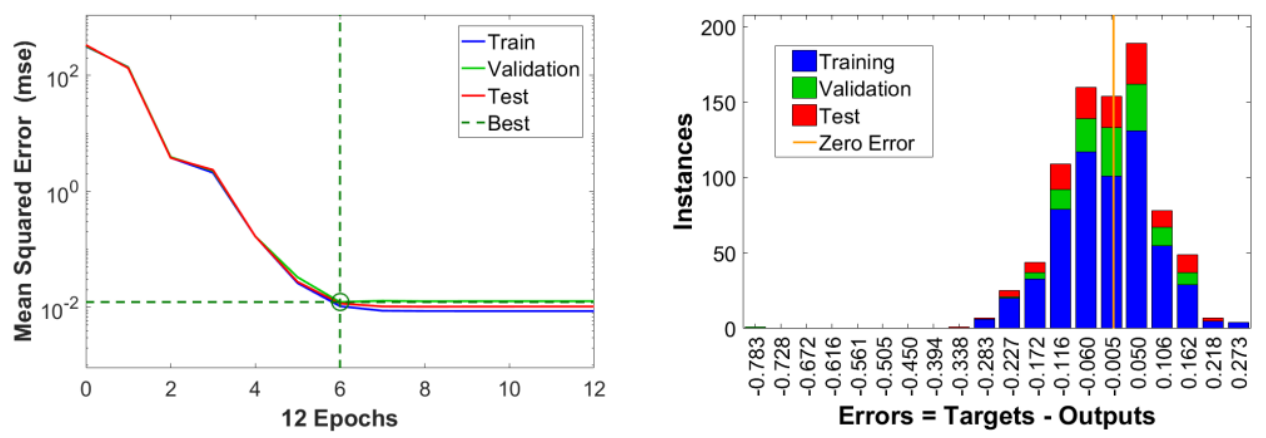

Figure 8: Neural Network Performance and Error Histogram. Left: Performance, best validation performance is 0.012388 at epoch 6. Right: Error histogram with 20 Bins.

\subsection{Voltage adjustment}

The output of the neural network is an offset voltage value which is used to characterize the jetting behavior. As it has the same unit and dimension with the control variable, it is easy to implement a control strategy like PID to adjust the drive voltage applied to the print head. A PID equation is

$$
\text { Output }=K_{P} \operatorname{err}(t)+K_{I} \int \operatorname{err}(t) d t+K_{D} \frac{d}{d t} \operatorname{err}(t) \text {, }
$$

where $\operatorname{err}(t)$ is the feedback value outputted from the neural network. To implement in a discretetime PID algorithm, the integration part is the sum of all the errors, and the derivative part is the difference between current error and previous error divided by time period. As the system response requirement is at the level of seconds, after tuning the PID parameters in our control system, a proportional control is good enough to achieve a stable jetting behavior.

\section{Results}

In this section, we first use our feature extraction method to understand the behavior of the LMJP process, and then we describe a case study to demonstrate the feedback control system.

\subsection{Experimental study}

A feedback control system is to adjust the inputs parameters based on the current status to compensate the defects in subsequent processes, and thus it is based on an assumption that the process is repeatable or at least stable in a short period. To show that the jetting process with the current setup is repeatable, we have conducted an experiment with a typical piezo nozzle under the working situation: applied voltage $50 \mathrm{~V}$, pressure $-10 \mathrm{mmH}_{2} \mathrm{O}$, jetting frequency $300 \mathrm{~Hz}$, and this test lasted for approximately 40 minutes with 300 times of jetting. Image capturing and feature extraction are done for each jet, and the extracted features are recorded which are plotted in Figure 9. The plots show quite consistent results over time with only a little variation, which is mostly caused by the varying environment and possibly the hardware. The standard deviations of satellite, ligament, volume and speed are $0 \%, 2.0 \%, 0.8 \%$ and $0.77 \%$ to the mean values, respectively. This experiment indicates that the jetting behavior and process are stable. 

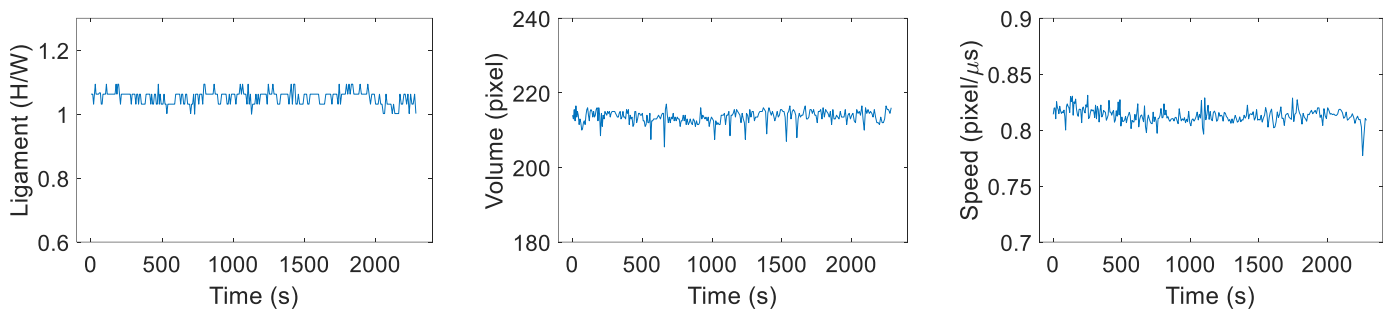

Figure 9: Repeatability test results. The plot for satellite is not shown as it is just a straight line.

In process modeling, we know that there are independent variables (controllable) and dependent variables (uncontrollable), but there are also unknown unknowns - the ones we do not know we do not know (e.g., the environment light may affect the jetting behavior, however, the relation between them as well as the existence of such fact itself is unknown to us). To account for the dependent variables and unknown unknowns as much as possible, the independent variables have to be significant enough that can provide a wide range of available adjustments to the output results. To have a better understanding of the variables in the LMJP process and the jetting behavior, we conduct another experiment to study the effect of parameters. Although it is impossible to test for unknown unknowns, the relationship between variables can still be found with those knowns. Two variables in LMJP are used here: voltage and pressure. In practice, the consumption of material, the changes of temperature and atmosphere all have influences on the pressure, but we do our best to keep all these variables consistent in this experiment. The experiment is performed with the typical working range of the voltage from $35 \mathrm{~V}$ to $60 \mathrm{~V}$ and the pressure applied from $-30 \mathrm{mmH}_{2} \mathrm{O}$ to $0 \mathrm{mmH}_{2} \mathrm{O}$. The results are shown in Figure 10. It can be seen that both of the parameters have effects on the jetting behavior indicated by the extracted features, but the change in voltage gives a level of difference while the change in pressure just gives a relatively mild influence. For example, with the increase of voltage from $35 \mathrm{~V}$ to $60 \mathrm{~V}$ under the pressure of $-30 \mathrm{mmH}_{2} \mathrm{O}$, the ligament is increased from 1 to 2.5 (250\%); but the ligament is just slightly increased from 1 to $1.2(20 \%)$ when the pressure is increased from -30 to $0 \mathrm{mmH}_{2} \mathrm{O}$ for voltage is $35 \mathrm{~V}$. Therefore, for the sake of effectiveness and efficiency, our control system adjusts the voltage level to compensate the random and unknown deflects. Nevertheless, there are exceptions such as when the pressure is changed from -20 to $-15 \mathrm{mmH}_{2} \mathrm{O}$ for voltage is $60 \mathrm{~V}$, the volume has a dramatic increase, which could result in the jetting behavior that cannot be corrected by the adjustments in voltage. Similar situations may also happen due to the unknown unknowns. For this kind of effects that cannot be compensated by parameter adjustment, it is detected and can only be removed by hardware modification. 

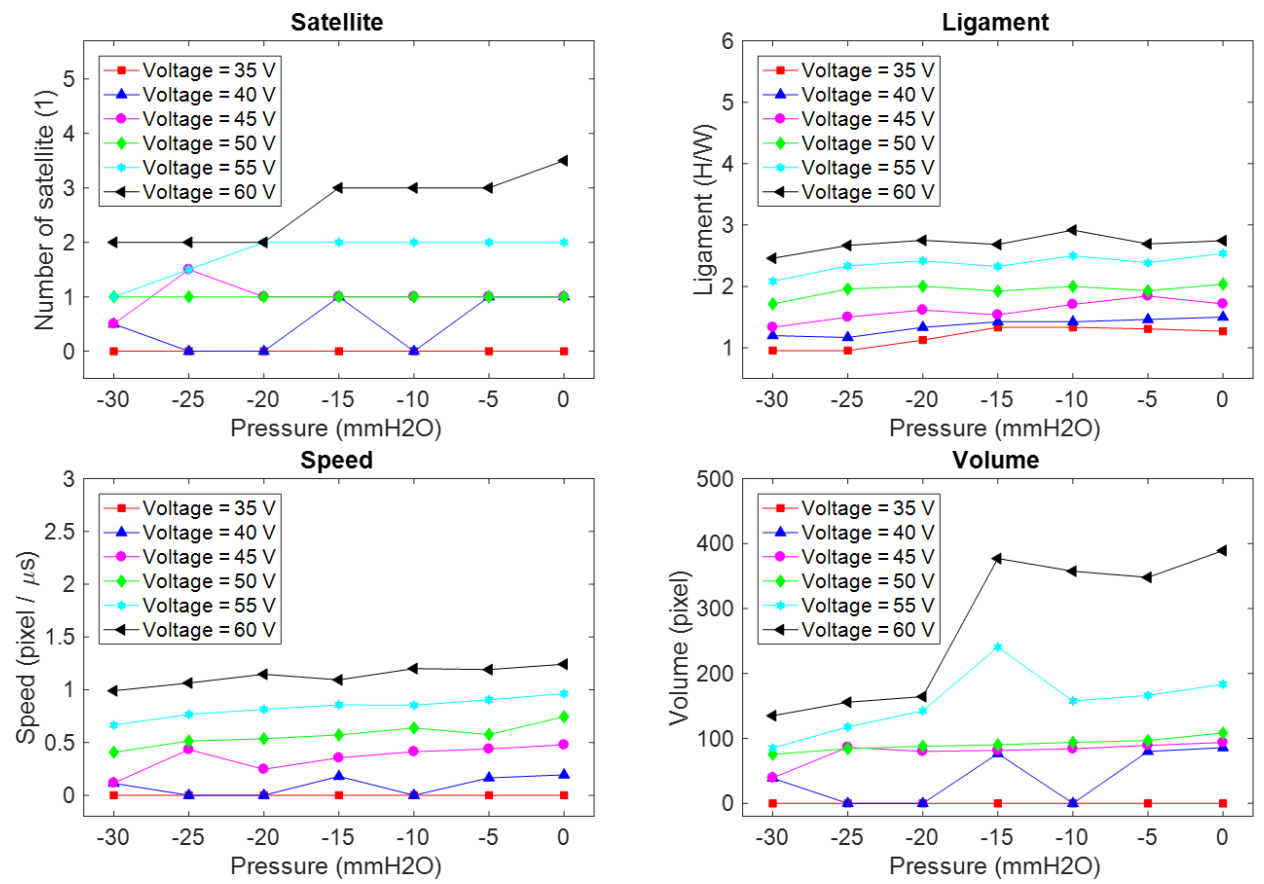

Figure 10: Experiments results with varying voltages and pressures.

\subsection{Case study}

Abnormal jetting behavior stabilization. The first experimental test case is carried out to verify the effectiveness and efficiency of the proposed control system when jetting process drifting occurs. At the beginning, the jetting device is set to exhibit an undesired jetting behavior, and then the controller is engaged to stabilize the jetting process. The detailed process is shown in Figure 11.

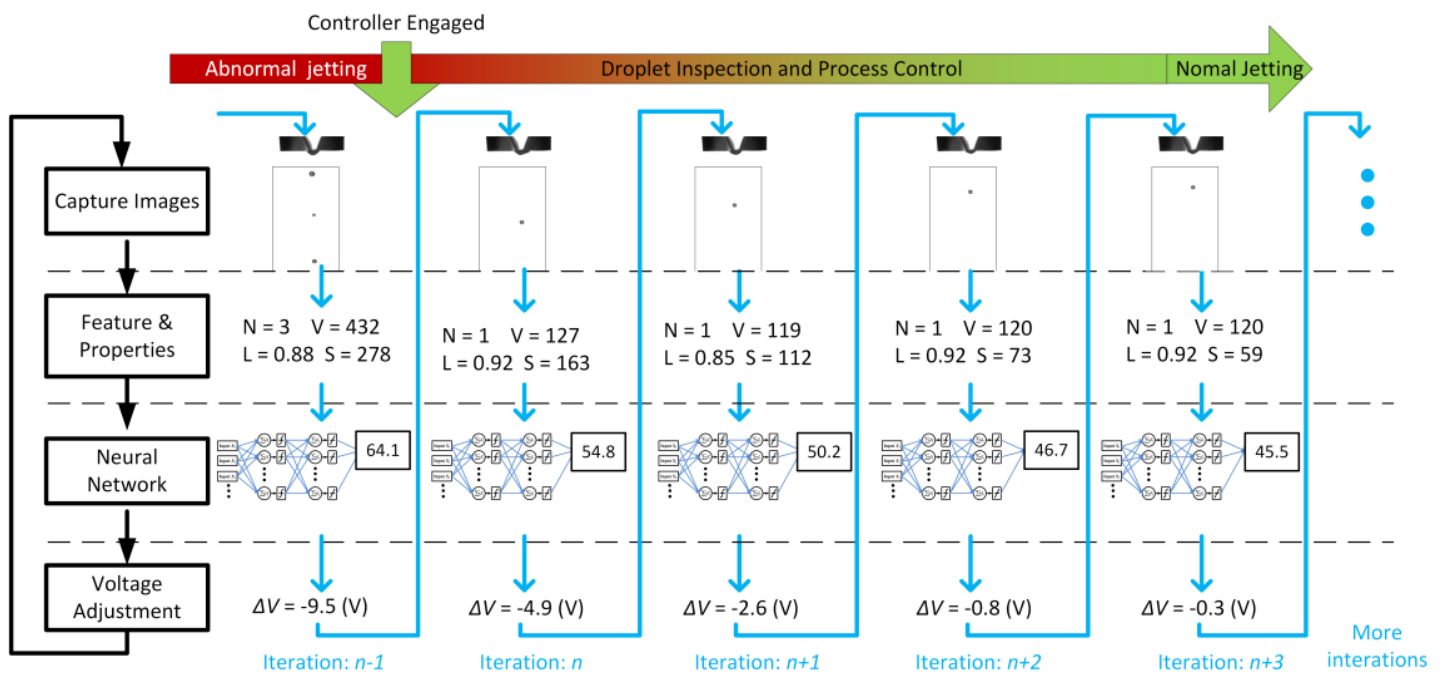

Figure 11: A case study for offset jetting stabilization 
First, images of the jetting process are captured under the piezo dispenser in each iteration. The images show instable droplets and undesirable satellites generated. After the vision inspection, features are extracted. In the iteration $n-1$, the system identifies the following features: satellite $(\mathrm{N})=$ 3 , ligament $(L)=0.88$, volume $(V)=432$, speed $(S)=278$. These features jointly describe the jetting behavior, through the neural network, they are mapped to a voltage value $64.1 \mathrm{~V}$. The control target is set based on images which give satellite $(N)=1$, ligament $(L)=1$, volume $(V)=120$, speed $(S)=60$. These features are mapped to a voltage value of $45 \mathrm{~V}$. When the controller is engaged, the virtual voltage $64.1 \mathrm{~V}$ is compared to the target value. After the PID process, a voltage adjustment of negative $9.5 \mathrm{~V}$ is applied to the printing head. Then the iteration continues, the system keeps monitoring the jetting behavior. The test case shows that the vision inspection can provide a proper adjustment of the drive voltage based on the captured images.

Uncontrollable disturbance compensation. The second test case is carried out to test the effectiveness of the controller when some uncontrollable variable changes during the jetting process. In this test case, the pressure applied to the jetting material decreased which disturbs the jetting process. Even though the pressure is not controlled by the proposed system, the drive voltage is adjusted by the controller to compensate the disturbing and get a stable desired jetting behavior. The detailed process is shown in Figure 12.

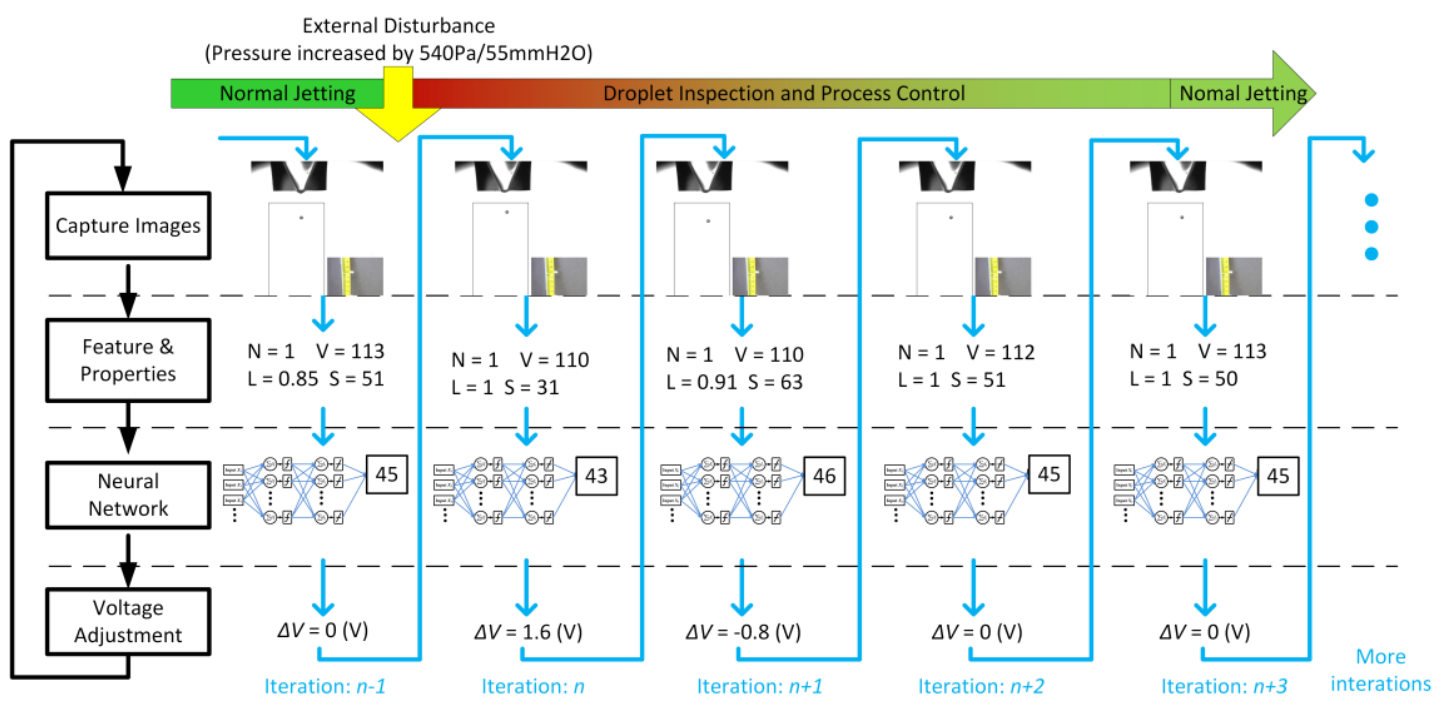

Figure 12 A case study for uncontrollable disturbing compensation

During the printing process, the pressure decreasing caused the jetting behavior changing. The features extracted from the images show that the droplet volume and speed decreased from iteration $n$ $l$ to iteration $n$. Through the neural network, this change can also be reflected from the virtual voltage changing from $45 \mathrm{~V}$ to $43 \mathrm{~V}$. With the PID control, the drive voltage was increased by $1.6 \mathrm{~V}$ for the next droplet. After several iterations, the jetting behavior is restored to the same situation as before the disturbance is applied. This test case shows that even the jetting condition is changed by external disturbance, the controller can successfully adjust the applied voltage to correct the jetting behavior. It should be noted that the monitoring and control process, in general, takes less than one second, which is much faster than the fluctuation of uncertain influences such as pressure changes caused by material consumption. The detailed in-situ droplet inspection and control process is shown in the following video: http://www.acsu.buffalo.edu/ chizhou/video/LMJP_Simulation.mp4. 


\section{Conclusions}

In this paper, we present a novel online monitoring and in-situ correction framework for inkjetbased 3D printing system. This framework seamlessly integrates efficient image processing techniques and machine learning technology to solve complex control problem. The central hypothesis of the proposed technique is that the random and uncontrollable process variations can be compensated by controllable and deterministic design variables. The major components of the proposed system include the vision-based droplet feature extraction, neural network and PID control subsystems. The output of the system is the optimized voltage level which is used to control the droplet jetting behaviour. In the proposed approach, a sequence of dynamic images for the droplet are captured by a CCD camera, and four properties (satellite, ligament, volume, and speed) of the droplet are extracted from both frozen and dynamic images to quantify the droplet behaviour. A neural network is proposed to identify the voltage change, which is then feedback to the current voltage. To verify the effectiveness and efficiency of the proposed approach, we implement the system on a piezoelectric inkjet-based emulator. The test results show that the proposed closed-loop control system can achieve much more stable jetting behaviour, and the process cycle for each droplet takes less than 0.5 seconds. Therefore, the proposed approach is promising to achieve online monitoring and in-situ correction for jetting based 3D printing processes.

In the future, we will extend the current prototype module from emulator to in-field LMJP machine. We will incorporate parallel computation techniques to further boost the online processing speed and feature extraction accuracy. In our current setup, we only considered the amplitude of the waveform as the system output, but we will consider other important parameters such the dwell/echo time of the waveform, the pressure/vacuum level, and environment temperatures. The significance of the extracted features on relationships with different types of droplet dynamics in the nonlinear model will also be investigated, where the collected feature values will be presented in a feature space and clustering will be applied to learn the correlation. It should be noted that the same strategy can be extended to the new process parameters with trivial effort.

\section{Acknowledgements}

This material is based upon work partially supported by the Center of Excellence in Materials Informatics and the Natural Sciences \& Engineering Research Council of Canada (NSERC) grant \# RGPIN-2017-06707. Any opinions, findings, and conclusions or recommendations expressed in this material are those of the authors and do not necessarily reflect the views of the agencies.

\section{References}

[1] Wei Gao, Yunbo Zhang, Devarajan Ramanujan, Karthik Ramani, Yong Chen, Christopher B Williams, Charlie CL Wang, Yung C Shin, Song Zhang, and Pablo D Zavattieri, "The status, challenges, and future of additive manufacturing in engineering," Computer-Aided Design, vol. 69, pp. 65-89, 2015.

[2] Terry Wohlers, "additive manufacturing and 3D printing State of the industry," Wohlers Associates, Fort Collins, CO, 2013.

[3] IDTechEx Ltd, "3D Printing of Metals 2015-2025 :Pricing, properties and projections for 3D printing equipment, materials and applications," March 2016.

[4] Robert Bogue, "3D printing: the dawn of a new era in manufacturing?," Assembly Automation, vol. 33, pp. 307-311, 2013. 
[5] Alexandru Pîrjan and Dana-Mihaela Petrosanu, "The impact of 3D printing technology on the society and economy," Journal of Information Systems \& Operations Management, p. 1, 2013.

[6] Lawrence E Murr, Edwin Martinez, Krista N Amato, Sara M Gaytan, Jennifer Hernandez, Diana A Ramirez, Patrick W Shindo, Frank Medina, and Ryan B Wicker, "Fabrication of metal and alloy components by additive manufacturing: examples of 3D materials science," Journal of Materials Research and technology, vol. 1, pp. 42-54, 2012.

[7] David L Bourell, Ming C Leu, and David W Rosen, "Roadmap for additive manufacturing: identifying the future of freeform processing," The University of Texas at Austin, Austin, TX, 2009.

[8] Vader System. http://vadersystems.com/.

[9] IH Karampelas, V Sukhotskiy, G Garg, A Verma, M Tong, S Vader, Z Vader, and EP Furlani, "Drop-on-Demand 3D Metal Printing," presented at the Proceedings of Nanotech Conference, Informatics, Electronics and Microsystems.

[10] John W Priest, Charles Smith, and Patrick DuBois, "Liquid metal jetting for printing metal parts," in Solid Freeform Fabrication Proceedings, University of Texas at Austin, TX, 1997, pp. 1-10.

[11] Melissa Orme and Robert F Smith, "Enhanced aluminum properties by means of precise droplet deposition," Journal of Manufacturing Science and Engineering, vol. 122, pp. 484493, 2000.

[12] Y Huang, LJ Yang, XZ Du, and YP Yang, "Finite element analysis of thermal behavior of metal powder during selective laser melting," International Journal of Thermal Sciences, vol. 104, pp. 146-157, 2016.

[13] Seyeon Hwang, Edgar I Reyes, Kyoung-sik Moon, Raymond C Rumpf, and Nam Soo Kim, "Thermo-mechanical characterization of metal/polymer composite filaments and printing parameter study for fused deposition modeling in the 3D printing process," Journal of Electronic Materials, vol. 44, pp. 771-777, 2015.

[14] Mary E. Kinsella, "Additive Manufacturing Workshop: Results and Plans," Airforce Research Laboratory, Dayton, OH 2009.

[15] Victoria Petrova, Advances in Engineering Research vol. 7. New York, NY: Nova Research, 2013.

[16] Y Huang and MC Leu, "Frontiers of Additive Manufacturing Research and EducationReport of NSF Additive Manufacturing Workshop," 2014.

[17] Yong Huang, Ming C Leu, Jyoti Mazumder, and Alkan Donmez, "Additive Manufacturing: Current State, Future Potential, Gaps and Needs, and Recommendations," Transactions of the ASME, Journal of Manufacturing Science and Engineering, vol. 137, p. 014001, 2015.

[18] J Mazumder, D Dutta, N Kikuchi, and A Ghosh, "Closed loop direct metal deposition: art to part," Optics and Lasers in Engineering, vol. 34, pp. 397-414, 2000.

[19] Dongming Hu and Radovan Kovacevic, "Sensing, modeling and control for laser-based additive manufacturing," International Journal of Machine Tools and Manufacture, vol. 43, pp. 51-60, 2003.

[20] Ehsan Toyserkani and Amir Khajepour, "A mechatronics approach to laser powder deposition process," Mechatronics, vol. 16, pp. 631-641, 2006.

[21] D Salehi and M Brandt, "Melt pool temperature control using LabVIEW in Nd: YAG laser blown powder cladding process," The international journal of advanced manufacturing technology, vol. 29, pp. 273-278, 2006.

[22] Matthias Faes, Wim Abbeloos, Frederik Vogeler, Hans Valkenaers, Kurt Coppens, and Eleonora Ferraris, "Process monitoring of extrusion based 3D printing via laser scanning," in PMI 2014 Conference Proceedings, 2014, pp. 363-367. 
[23] Yuan Cheng and Mohsen A Jafari, "Vision-based online process control in manufacturing applications," IEEE Transactions on Automation Science and Engineering, vol. 5, pp. 140$153,2008$.

[24] Bilal Hijazi, Thomas Decourselle, Sofija Vulgarakis Minov, David Nuyttens, Frederic Cointault, Jan Pieters, and Jürgen Vangeyte, The use of high-speed imaging systems for applications in precision agriculture: InTech, 2012.

[25] Christian Pfeifer, Dietmar Kuhn, and Andreas G Class, "Coupled measurement of droplet size distribution and velocity distribution in a fuel spray with digital imaging analysis under elevated pressure," in 15th International symposium on applications of laser techniques to fluid mechanics. Lisboa, Portugal:[sn], 2010.

[26] Kye-Si Kwon, "Speed measurement of ink droplet by using edge detection techniques," Measurement, vol. 42, pp. 44-50, 2009.

[27] MG Cabezas, A Bateni, JM Montanero, and AW Neumann, "A new drop-shape methodology for surface tension measurement," Applied surface science, vol. 238, pp. 480-484, 2004.

[28] JB Blaisot and J Yon, "Droplet size and morphology characterization for dense sprays by image processing: application to the Diesel spray," Experiments in fluids, vol. 39, pp. 977994, 2005.

[29] Kai Xu and Yong Chen, "Photocuring Temperature Study for Curl Distortion Control in Projection-Based Stereolithography," Journal of Manufacturing Science and Engineering, vol. 139, p. 021002, 2017.

[30] Kai Xu, Tsz-Ho Kwok, Zhengcai Zhao, and Yong Chen, "A Reverse Compensation Framework for Shape Deformation Control in Additive Manufacturing," Journal of Computing and Information Science in Engineering, vol. 17, p. 021012, 2017.

[31] Oren Breslouer, "Rayleigh-Plateau Instability: Falling Jet," Project Report, Princeton University, 2010.

[32] Mitja Blazincic, "Physics of Ink-jet Printing," 2008.

[33] Chi Zhou and Yong Chen, "Three-dimensional digital halftoning for layered manufacturing based on droplets," Transactions of the North American Manufacturing Research Institution of SME, vol. 37, pp. 175-182, 2009.

[34] Ted M Smith and Russell E Winstead, "Electrodynamic pump for dispensing molten solder," ed: Google Patents, 1995.

[35] Charles V Smith, John W Priest, and Patrick N DuBois, "Heat-resistant broad-bandwidth liquid droplet generators," ed: Google Patents, 1996.

[36] Jung-Hoon Chun, Christian H Passow, and Nam P Suh, "Droplet-based manufacturing," CIRP ANNALS-manufacturing technology, vol. 42, pp. 235-238, 1993.

[37] Melissa E Orme and Eric P Muntz, "Method and apparatus for droplet stream manufacturing," ed: Google Patents, 1993.

[38] Donald J Hayes, Michael T Boldman, and David B Wallace, "Method and apparatus for dispensing spherical-shaped quantities of liquid solder," ed: Google Patents, 1993.

[39] CV Smith Jr, "Analysis ofDroplet Formation in Ink Jet Printers," Unpublished Research, The University ofTexas at Arlington, 1974.

[40] Kye-Si Kwon, Jung-Kook Go, Jin-Won Kim, and Dongho Oh, "In Situ Measurement of Instantaneous Jetting Speed Curve," in NIP \& Digital Fabrication Conference, 2010, pp. 1822.

[41] Li Erqiang, "The Generation And Experimental Study Of Microscale Droplets In Drop-OnDemand Inkjet Printing," 2010.

[42] Nobuyuki Otsu, "A threshold selection method from gray-level histograms," Automatica, vol. 11, pp. 23-27, 1975.

[43] Pierre Soille, Morphological image analysis: principles and applications: Springer Science \& Business Media, 2013. 